\title{
Statistical Analysis of Amir Schoor's Algorithm in Sequential and Parallel Environment
}

\author{
Rohit Kumar $^{1}$, Amritanjali ${ }^{1}$, Soubhik Chakraborty ${ }^{2, *}$ \\ ${ }^{1}$ Depatment of Computer Science and Engineering, Birla Institute of Technology, Mesra, Ranchi-835215, Jharkhand, India. \\ ${ }^{2}$ Department of Mathematics, Birla Institute of Technology, Mesra, Ranchi-835215, Jharkhand, India.
}

How to cite this paper: Rohit Kumar, Amritanjali, Soubhik Chakraborty. (2021) Statistical Analysis of Amir Schoor's Algorithm in Sequential and Parallel Environment. Journal of Applied Mathematics and Computation, 5(3), 171-186.

DOI: $10.26855 /$ jamc.2021.09.004

Received: July 20, 2021

Accepted: August 16, 2021

Published: September 3, 2021

*Corresponding author: Soubhik Chakraborty, Department of Mathematics, Birla Institute of Technology, Mesra, Ranchi-835215, Jharkhand, India.

Email: soubhikc@yahoo.co.in

\begin{abstract}
Matrix multiplication is an essential constituent of most of the computer models and systems implementing Numerical Algorithms, Signal and image processing, Graph theory, Digital control. So reduction of multiplication time can influence drastically the overall system performance. This paper gives a statistical analysis of average time complexity for matrix multiplication with two dense square matrices of order ' $\mathrm{n}$ ' and achieves an empirical $\mathrm{O}_{\text {emp }}\left(\mathrm{n}^{2}\right)$ complexity in sequential and $\mathrm{O}_{\text {emp }}(\mathrm{n})$ complexity in parallel computing environment with Amir Schoor's Algorithm using different probability distributions characterizing the matrix elements. Amir Schoor's algorithm, although initially developed for sparse matrices, also performs well for dense matrices for two reasons. First, it is faster to work with rows only (as in Schoor's algorithm) than to work with both rows and columns (as in classical matrix multiplication). Secondly, as we have shown, the $\mathrm{n}^{2}$ comparisons turn out to be "heavier" (in terms of weight, with time of an operation being considered as its weight) than the $\mathrm{O}\left(\mathrm{d}_{1} \mathrm{~d}_{2} \mathrm{n}^{3}\right)$ multiplications on the average, wherein lies the concept of a weight based statistical bound whose empirical estimate over a finite feasible range is empirical $\mathrm{O}_{\text {emp }}\left(\mathrm{n}^{2}\right)$ in our case. Here $\mathrm{d}_{1}$ and $\mathrm{d}_{2}$ are the densities of the pre and post factor matrices.
\end{abstract}

\section{Keywords}

Statistical, Amir Schoor's Algorithm, Sparse Matrices, Dense Matrices, Average Case Complexity, Matrix Multiplications, Probability Distribution

\section{Introduction}

Amir Schoor's Algorithm is a fast algorithm for the multiplication of two sparse matrices, whose average time complexity is $O\left(d_{1} d_{2} n^{3}\right)$ [1] where $d_{1}$ and $d_{2}$ are the densities of the pre-factor and post-factor matrices and it has already been stated before that if the product of these densities is kept at $1 / n$, we can trivially get an empirical $O\left(n^{2}\right)$ [2] complexity in sequential execution over continuous uniform probability distribution characterizing the matrix elements.

This paper presents how we can further reduce the average time complexity to an empirical $\mathrm{O}(\mathrm{n})$ for matrix multiplication in parallel execution (shared-memory system). We would also be ensuring if empirical $\mathrm{O}\left(\mathrm{n}^{2}\right)$ complexity for matrix multiplication of two dense matrix still holds for sequential execution even after subjecting the matrices over different probability distributions characterizing their elements applying Amir Schoor's Algorithm.

For our study, we would be using statistical analysis [3] where the CPU time of algorithm is used as the weight [4] of a computing operation which is the corresponding time taken for its execution.

Further using "Mintab" [5] statistics package we will be observing the fits to quadratic and linear plots for the outputs of both sequential and parallel implementation to get to our conclusion. 


\section{Algorithm Implementation}

\subsection{Amir Schoor's Algorithm}

Let us assume 'A', 'B', and 'C' be pre-factor, post-factor and product matrices respectively. Then Amir Schoor's algorithm states that for every non-zero element $a(i, k)$ of pre-factor matrix ' $A$ ', multiply the $k^{\text {th }}$ row of post-factor matrix 'B' by element $a(i, k)$ and add it to the $i^{\text {th }}$ row of product matrix ' $\mathrm{C}$ ' .

Although Amir Schoor's Algorithm was devised for multiplication of sparse matrices, we would be using it for multiplication of dense matrices where pre-factor matrix is kept as dense as triangular matrix with density $(n+1) /(2 n)[6]$ and post-factor matrix is kept fully dense with density 1 . We have initialized the product matrix with all zero entries for their elements before execution of the algorithm.

As a triangular matrix is dense with density $(n+1) /(2 n)$ and there is no specific density defined at which the matrix changes from sparse to dense, we would agree to refer the pre-factor matrix as dense matrix in which the fraction of zeroes is approximately equal to that in a triangular matrix.

Since the computer program for dense matrices would be used here rather than the one for sparse matrices, hence we need not use Amir Schoor's algorithm's original data structure, i.e., the structure normally used for multiplication of sparse matrices where our concern is the number of non-zero elements in the sparse matrix and not its size.

Matrix elements of pre and post-factor matrices are generated using two continuous probability distributions namely normal distribution and exponential distribution and two discrete probability distributions namely Poisson distribution and Binomial distribution.

For Normal distribution the value of $\mu$ (mean) is set to 3.0 and $\sigma$ (standard deviation) is set to 4.0, for Exponential distribution the value of $\lambda$ (rate parameter) is set to 4.0 , for Binomial distribution the value of $\mathrm{n}$ (number of trials) is set to 5.0 and $p$ (success probability in each trial) is set to 0.3 and for Poisson distribution value of $\lambda$ (event rate) is set to 7.0 .

The program for both sequential and parallel implementations [7, 8] are executed on Windows 10 Pro 64-bit OS with Intel i5-2450M CPU having dual-core architecture running at 2.50GHz with 8192MB RAM and with each core of CPU running four threads.

\subsection{Sequential Implementation}

The program for sequential implementation is written in $\mathrm{C}++11$ to calculate the execution time (in seconds) of Amir Schoor's algorithm for each probability distribution and the output is then used to generate the Regression plots using Minitab Statistics Package as Fitted line plots and Residual plots for each probability distribution.

The pseudo code of the Amir Schoor's Algorithm for sequential implementation is as follow:



For pre-factor matrix, zeros are assigned randomly using following condition:

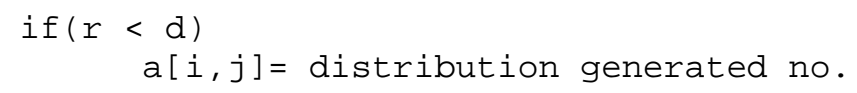


else

$a[i, j]=0$

where $d=\underline{(n+1)}$ and $r=\underline{\text { (distribution generated random no. })}$

$2 n \quad$ (maximum value of random no. generated)

\subsection{Parallel Implementation}

The parallel implementation is done in shared-memory architecture and program for parallel execution is written in C++11 using OpenMP (Open Multi-Processing) $[9,10]$ API (application programming interface) to calculate the execution time of algorithm for each probability distribution and the regression plots are generated using the output for each probability distribution.

The NUM_THREADS i.e. the number of threads is set to 4 as the CPU core is designed to run 4 threads per core.

Since we are using OpenMP, we don't need to specify the behaviour of each thread individually as each threads are assigned tasks by the compilar at the run time.

The pseudo code of the Amir Schoor's Algorithm for parallel implementation is as follow:

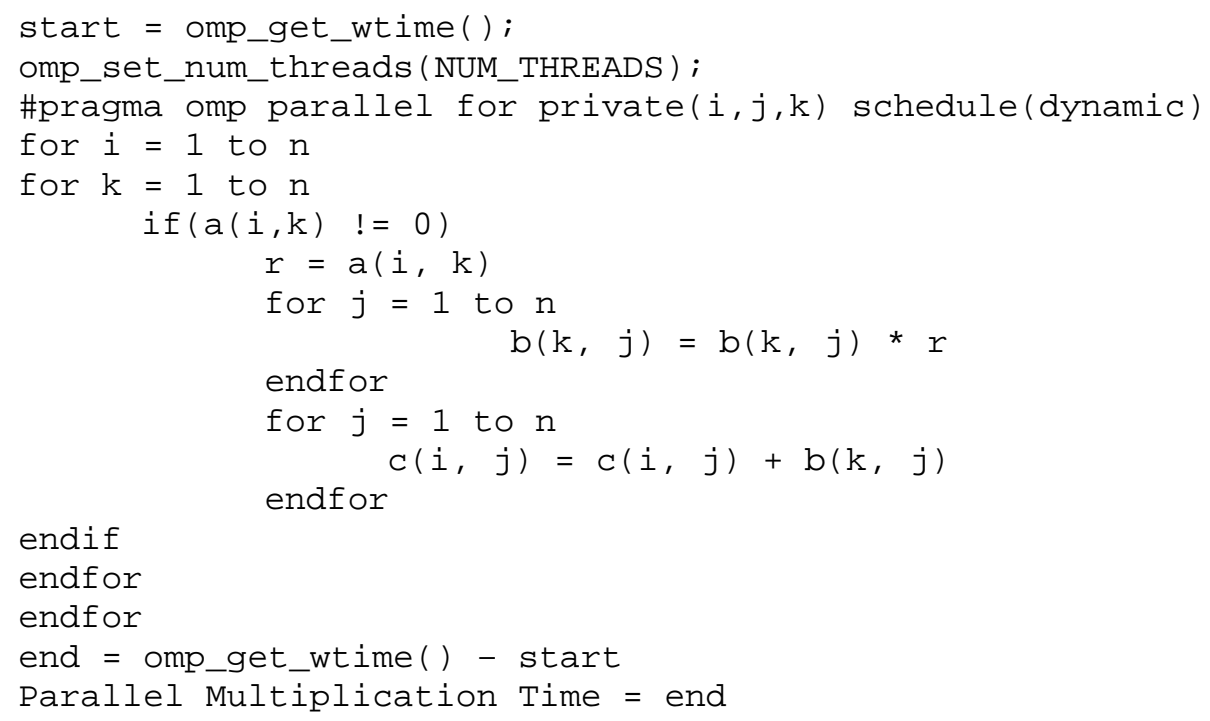

Here also, for pre-factor matrix, zeros are assigned randomly using the same condition as shown in sequential implementation.

\section{Results and Discussion}

The following points are noteworthy.

(1) In Fitted line plot, R-squared is a statistical measure of how close the data are to the fitted regression line. It is also known as the coefficient of determination, or the coefficient of multiple determination for multiple regression. Its value is always between 0 and $100 \%$. The higher the R-squared, the better the model fits your data.

(2) The adjusted R-squared is a modified version of R-squared that has been adjusted for the number of predictors in the model.

(3) ' $S$ ' is known both as the standard error of the regression and as the standard error of the estimate. It represents the average distance that the observed values fall from the regression line. Smaller values of ' $S$ ' are better because it indicates that the observations are closer to the fitted line.

(4) A Residual plot is a graph that is used to examine the goodness-of-fit in regression where a residual is the difference between an observed value and its corresponding fitted value.

(5) A histogram of the residuals shows the distribution of the residuals for all observations. It is used to determine whether the data are skewed or whether outliers exist in the data.

(6) Normal probability plot of residuals uses the normal plot of residuals to verify the assumption that the residuals are 
normally distributed.

(7) Residuals versus fits uses the residuals versus fits plot to verify the assumption that the residuals have a constant variance.

(8) Residuals versus order of data use the residuals versus order plot to verify the assumption that the residuals are uncorrelated with each other.

\subsection{Sequential Execution}

For the results generated by Normal, Exponential, Binomial and Poisson Distributions refer to Tables 1, 2, 3 and 4 respectively.

For the corresponding fitted line plots refer to Figures 1a, 2a, 3a and 4a and for the corresponding residual plots refer to Figures $1 \mathrm{~b}, 2 \mathrm{~b}, 3 \mathrm{~b}$ and $4 \mathrm{~b}$ respectively.

Table 1. Sequential Execution Time for Normal Distribution (in seconds)

\begin{tabular}{cccccccccccc}
\hline $\begin{array}{c}\text { Trials } \\
\mathbf{n}\end{array}$ & $\mathbf{1}$ & $\mathbf{2}$ & $\mathbf{3}$ & $\mathbf{4}$ & $\mathbf{5}$ & $\mathbf{6}$ & $\mathbf{7}$ & $\mathbf{8}$ & $\mathbf{9}$ & $\mathbf{1 0}$ & Avg. \\
\hline $\mathbf{2 2 0 0}$ & 0.015 & 0.015 & 0.016 & 0.015 & 0.017 & 0.015 & 0.014 & 0.017 & 0.018 & 0.016 & 0.0158 \\
$\mathbf{2 5 0 0}$ & 0.021 & 0.022 & 0.022 & 0.022 & 0.022 & 0.020 & 0.022 & 0.024 & 0.023 & 0.022 & 0.0220 \\
$\mathbf{2 8 0 0}$ & 0.027 & 0.026 & 0.025 & 0.025 & 0.028 & 0.024 & 0.025 & 0.028 & 0.027 & 0.024 & 0.0259 \\
$\mathbf{3 1 0 0}$ & 0.033 & 0.028 & 0.029 & 0.028 & 0.029 & 0.029 & 0.028 & 0.029 & 0.029 & 0.028 & 0.0290 \\
$\mathbf{3 4 0 0}$ & 0.034 & 0.033 & 0.034 & 0.033 & 0.033 & 0.036 & 0.033 & 0.034 & 0.036 & 0.033 & 0.0339 \\
$\mathbf{3 7 0 0}$ & 0.039 & 0.043 & 0.046 & 0.041 & 0.042 & 0.042 & 0.039 & 0.042 & 0.044 & 0.041 & 0.0419 \\
$\mathbf{4 0 0 0}$ & 0.046 & 0.046 & 0.048 & 0.045 & 0.047 & 0.046 & 0.046 & 0.046 & 0.050 & 0.048 & 0.0468 \\
$\mathbf{4 3 0 0}$ & 0.055 & 0.055 & 0.056 & 0.058 & 0.063 & 0.059 & 0.056 & 0.058 & 0.058 & 0.054 & 0.0572 \\
$\mathbf{4 6 0 0}$ & 0.063 & 0.064 & 0.071 & 0.062 & 0.063 & 0.070 & 0.063 & 0.064 & 0.064 & 0.074 & 0.0658 \\
$\mathbf{4 9 0 0}$ & 0.073 & 0.069 & 0.096 & 0.070 & 0.071 & 0.070 & 0.076 & 0.072 & 0.077 & 0.075 & 0.0749 \\
\hline
\end{tabular}

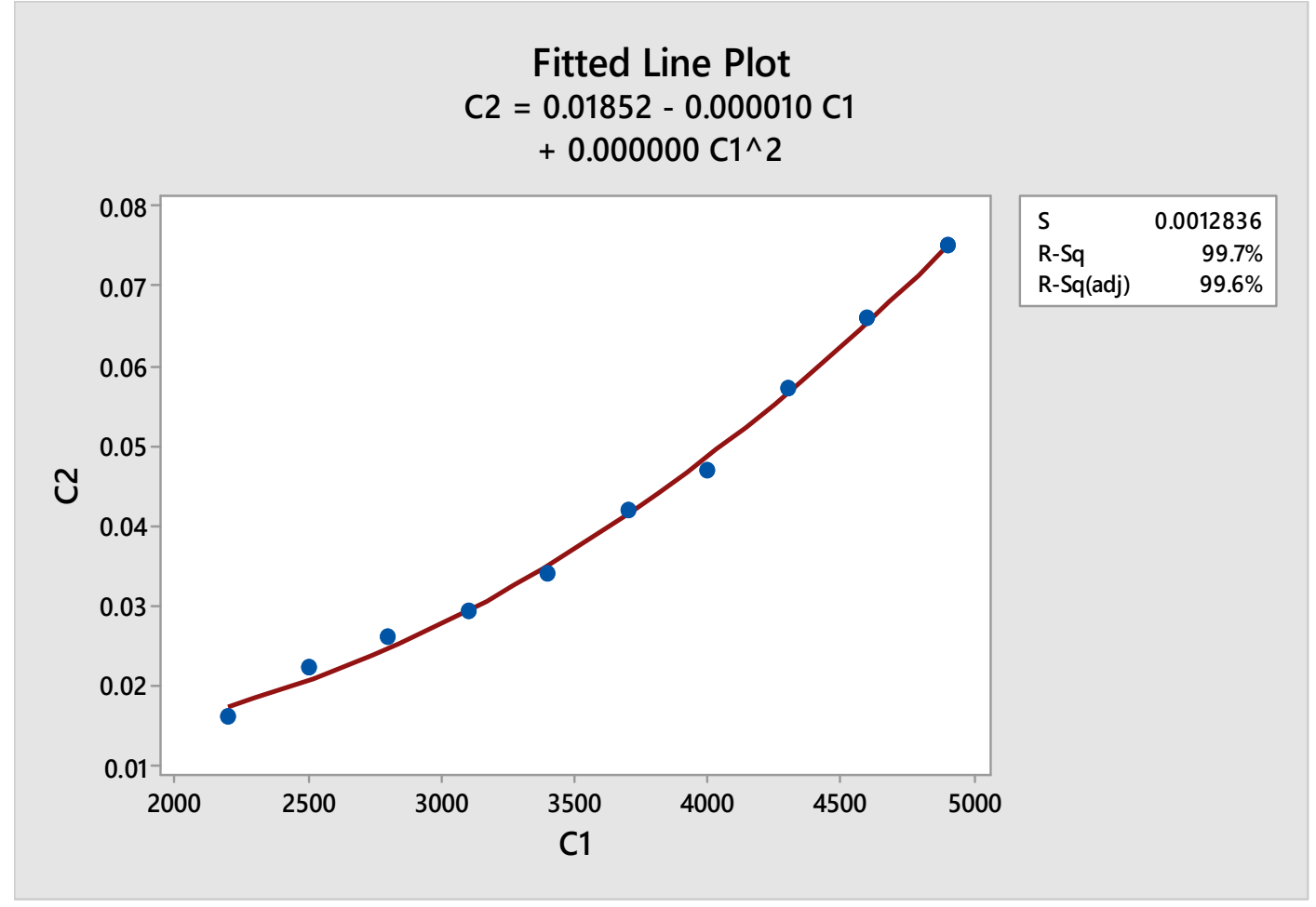

Figure 1a. Fitted line plot of Sequential Execution for Normal Distribution. 




Figure 1b. Residual plot of Sequential Execution for Normal Distribution.

Table 2. Sequential Execution Time for Exponential Distribution (in seconds)

\begin{tabular}{ccccccccccccc}
\hline $\begin{array}{c}\text { Trials } \\
\mathbf{n}\end{array}$ & $\mathbf{1}$ & $\mathbf{2}$ & $\mathbf{3}$ & $\mathbf{4}$ & $\mathbf{5}$ & $\mathbf{6}$ & $\mathbf{7}$ & $\mathbf{8}$ & $\mathbf{9}$ & $\mathbf{1 0}$ & $\mathbf{A v g .}$ \\
\hline $\mathbf{2 2 0 0}$ & 0.014 & 0.015 & 0.017 & 0.018 & 0.017 & 0.014 & 0.015 & 0.017 & 0.014 & 0.018 & 0.0159 \\
$\mathbf{2 5 0 0}$ & 0.018 & 0.024 & 0.021 & 0.021 & 0.025 & 0.020 & 0.022 & 0.018 & 0.022 & 0.021 & 0.0212 \\
$\mathbf{2 8 0 0}$ & 0.027 & 0.026 & 0.025 & 0.027 & 0.027 & 0.027 & 0.032 & 0.026 & 0.024 & 0.027 & 0.0268 \\
$\mathbf{3 1 0 0}$ & 0.028 & 0.029 & 0.029 & 0.028 & 0.027 & 0.029 & 0.028 & 0.029 & 0.029 & 0.028 & 0.0284 \\
$\mathbf{3 4 0 0}$ & 0.034 & 0.034 & 0.034 & 0.034 & 0.035 & 0.034 & 0.034 & 0.034 & 0.037 & 0.035 & 0.0345 \\
$\mathbf{3 7 0 0}$ & 0.039 & 0.047 & 0.040 & 0.041 & 0.048 & 0.039 & 0.041 & 0.045 & 0.044 & 0.044 & 0.0428 \\
$\mathbf{4 0 0 0}$ & 0.044 & 0.049 & 0.048 & 0.046 & 0.046 & 0.046 & 0.047 & 0.047 & 0.048 & 0.046 & 0.0467 \\
$\mathbf{4 3 0 0}$ & 0.061 & 0.053 & 0.059 & 0.053 & 0.055 & 0.062 & 0.055 & 0.055 & 0.057 & 0.055 & 0.0565 \\
$\mathbf{4 6 0 0}$ & 0.065 & 0.068 & 0.063 & 0.068 & 0.072 & 0.070 & 0.062 & 0.064 & 0.064 & 0.062 & 0.0658 \\
$\mathbf{4 9 0 0}$ & 0.070 & 0.070 & 0.084 & 0.070 & 0.075 & 0.072 & 0.070 & 0.077 & 0.074 & 0.072 & 0.0734 \\
\hline
\end{tabular}

On analyzing the fitted line plots of sequential implementation, we can certainly say that the observed data is perfectly fitting to the quadratic regression model as the value of R-squared and adjusted R-squared is above 99\% for each distribution with binomial distribution having the highest value as 99.8\% (see Figure 3a) and Poisson distribution having the lowest value as $99.5 \%$ (see Figure $4 a$ ). We can also observe that the value of ' $S$ ' is also very low each distribution. 




Figure 2a. Fitted line plot of Sequential Execution for Exponential Distribution.

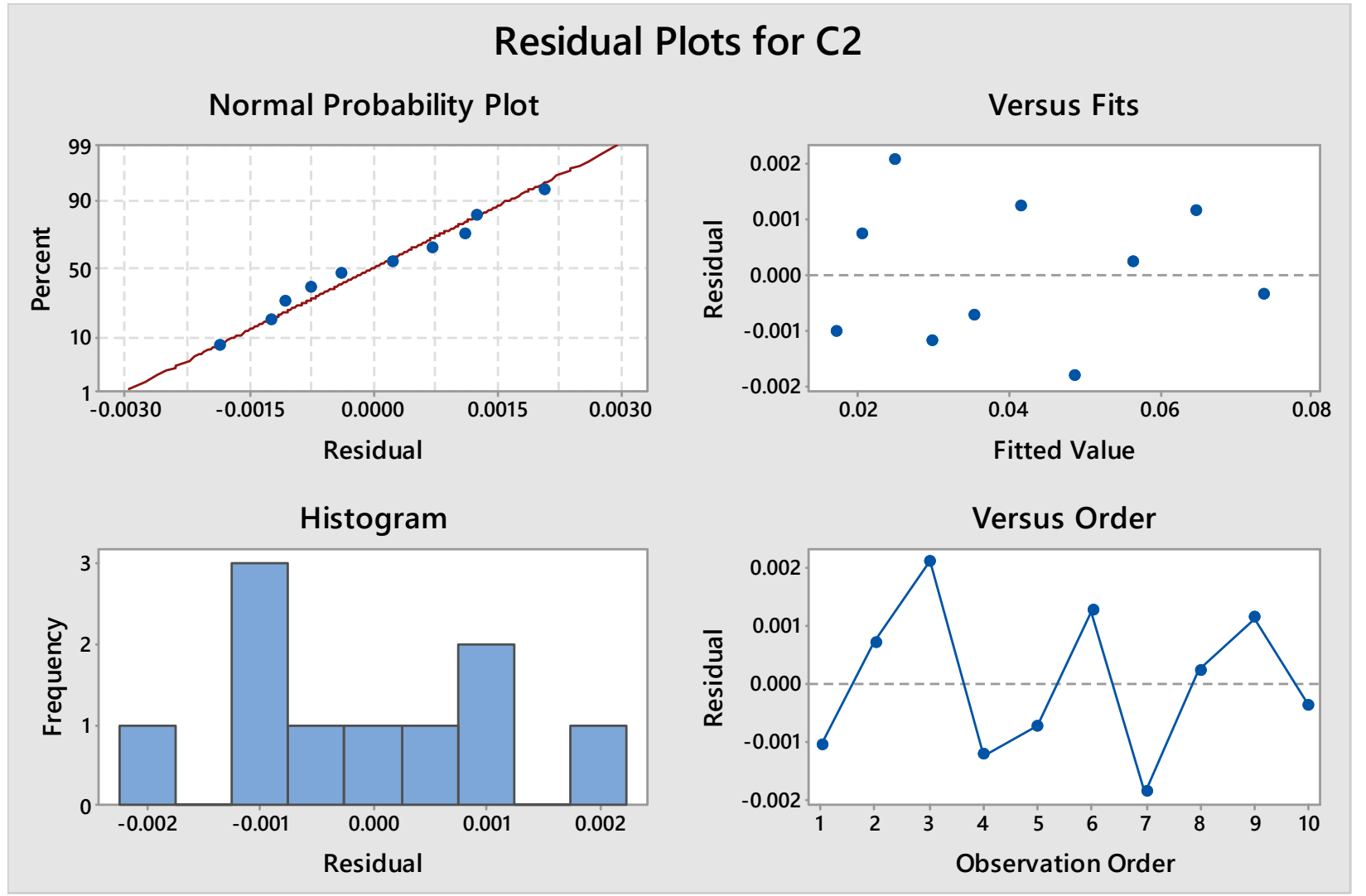

Figure 2b. Residual plot of Sequential Execution for Exponential Distribution. 
Table 3. Sequential Execution Time for Binomial Distribution (in seconds)

\begin{tabular}{ccccccccccccc}
\hline Trials & $\mathbf{1}$ & $\mathbf{2}$ & $\mathbf{3}$ & $\mathbf{4}$ & $\mathbf{5}$ & $\mathbf{6}$ & $\mathbf{7}$ & $\mathbf{8}$ & $\mathbf{9}$ & $\mathbf{1 0}$ & $\mathbf{A v g .}$ \\
$\mathbf{n}$ & & & & & & & & & & & & \\
\hline $\mathbf{2 2 0 0}$ & 0.014 & 0.017 & 0.017 & 0.014 & 0.017 & 0.017 & 0.015 & 0.014 & 0.017 & 0.017 & 0.0159 \\
$\mathbf{2 5 0 0}$ & 0.018 & 0.018 & 0.021 & 0.021 & 0.020 & 0.022 & 0.021 & 0.021 & 0.020 & 0.021 & 0.0203 \\
$\mathbf{2 8 0 0}$ & 0.026 & 0.026 & 0.025 & 0.026 & 0.022 & 0.025 & 0.026 & 0.025 & 0.026 & 0.025 & 0.0252 \\
$\mathbf{3 1 0 0}$ & 0.027 & 0.027 & 0.028 & 0.028 & 0.028 & 0.027 & 0.028 & 0.028 & 0.028 & 0.027 & 0.0276 \\
$\mathbf{3 4 0 0}$ & 0.034 & 0.033 & 0.033 & 0.033 & 0.038 & 0.033 & 0.034 & 0.034 & 0.038 & 0.033 & 0.0343 \\
$\mathbf{3 7 0 0}$ & 0.042 & 0.045 & 0.039 & 0.039 & 0.040 & 0.045 & 0.038 & 0.049 & 0.040 & 0.042 & 0.0419 \\
$\mathbf{4 0 0 0}$ & 0.046 & 0.049 & 0.059 & 0.046 & 0.050 & 0.045 & 0.047 & 0.048 & 0.046 & 0.048 & 0.0484 \\
$\mathbf{4 3 0 0}$ & 0.054 & 0.059 & 0.057 & 0.053 & 0.057 & 0.068 & 0.056 & 0.054 & 0.060 & 0.052 & 0.0570 \\
$\mathbf{4 6 0 0}$ & 0.063 & 0.079 & 0.062 & 0.062 & 0.067 & 0.065 & 0.061 & 0.062 & 0.059 & 0.063 & 0.0643 \\
$\mathbf{4 9 0 0}$ & 0.069 & 0.074 & 0.072 & 0.069 & 0.069 & 0.070 & 0.070 & 0.093 & 0.073 & 0.076 & 0.0735 \\
\hline
\end{tabular}

Fitted Line Plot

$$
\begin{aligned}
\mathrm{C} 2= & 0.01037-0.000006 \mathrm{C} 1 \\
& +0.000000 \mathrm{C} 1^{\wedge} 2
\end{aligned}
$$

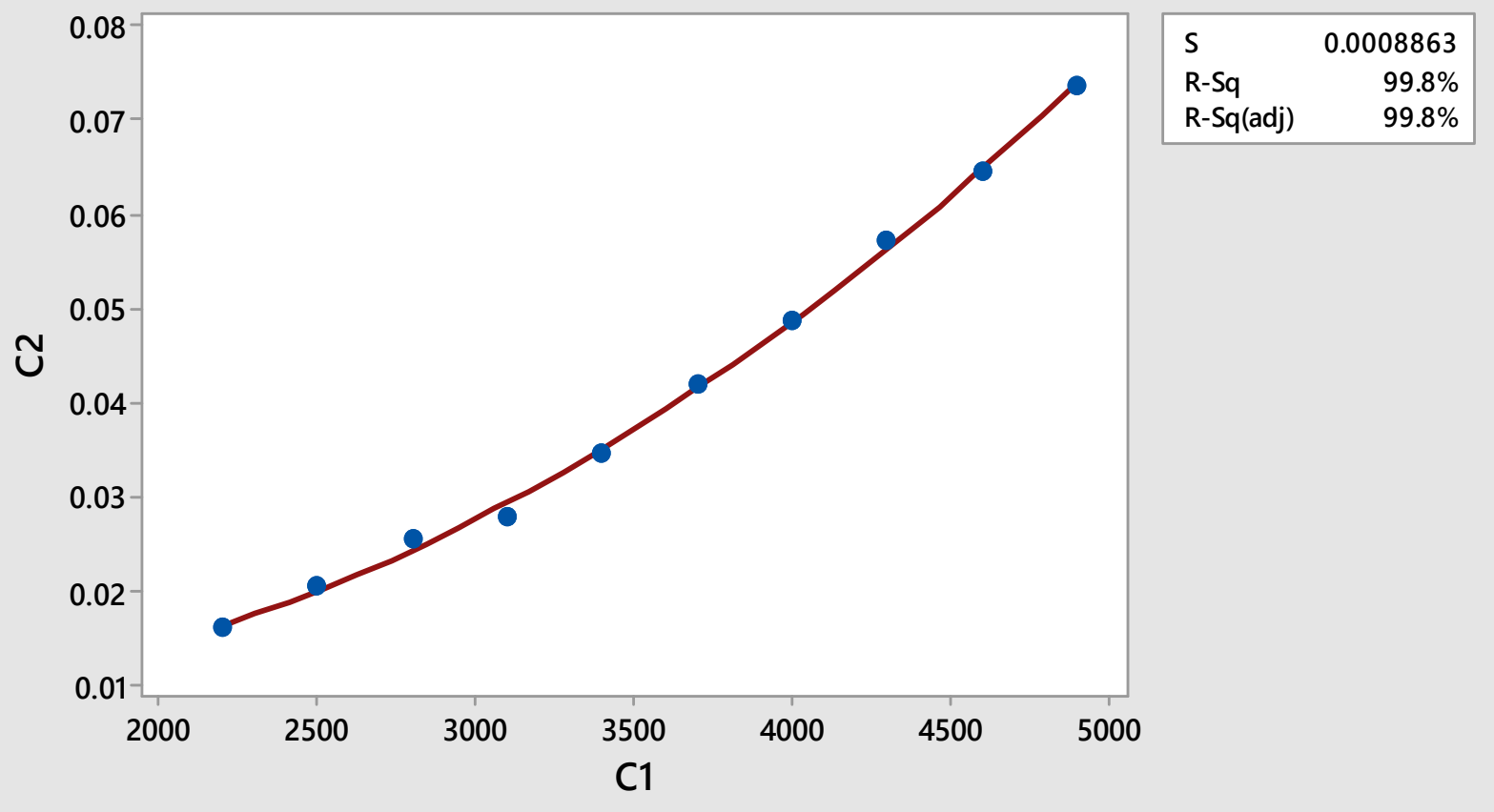

Figure 3a. Fitted line plot of Sequential Execution for Binomial Distribution.

On observing the Residual plots, we can analyze that the Normal probability plot for each distribution is almost a straight line except for Binomial distribution which suggest that residuals are normally distributed for each distribution except for Binomial distribution (see Figure 3b) with a point far away from the tail showing an outlier. 


\section{Residual Plots for C2}
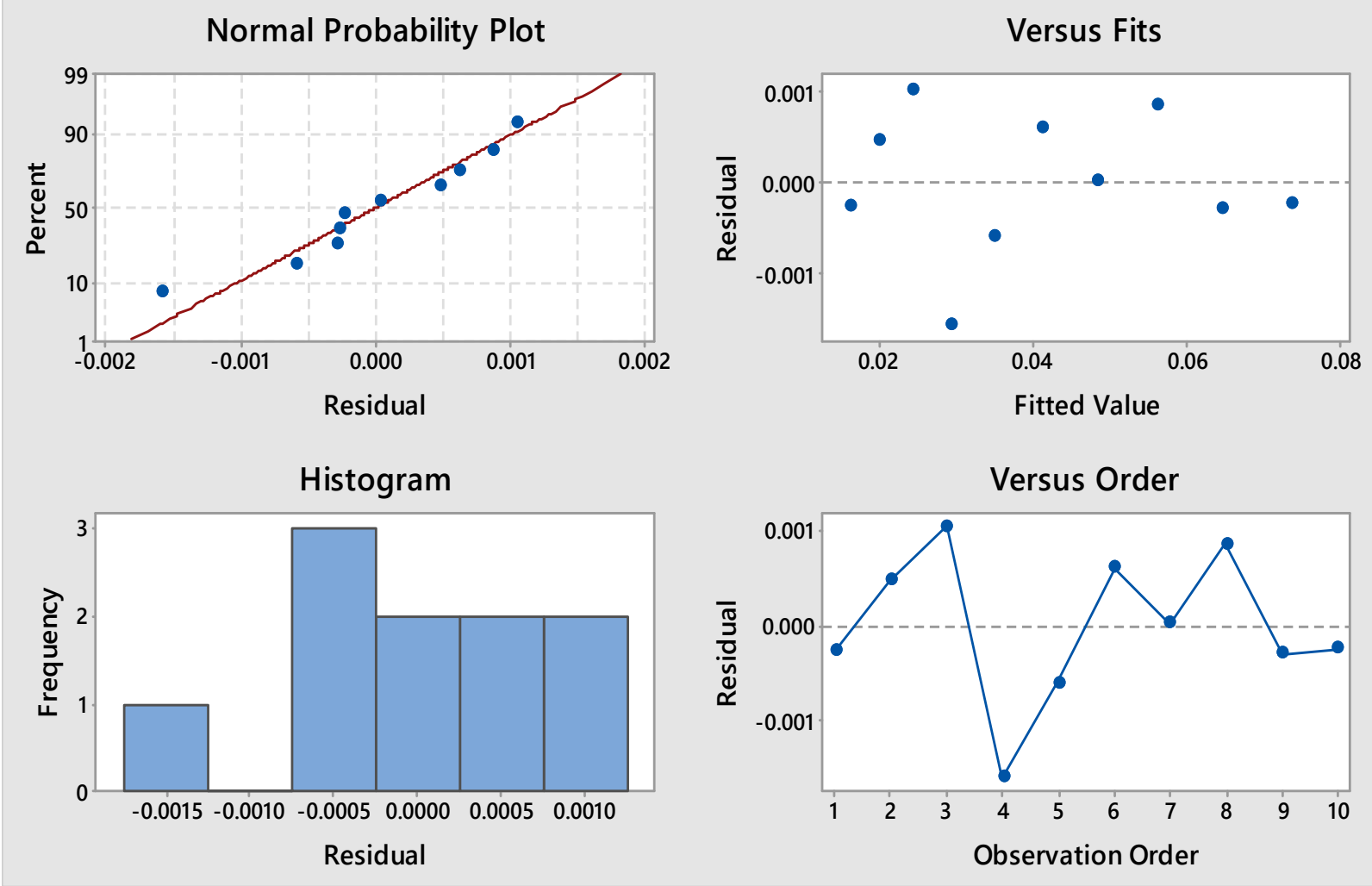

Figure 3b. Residual plot of Sequential Execution for Binomial Distribution.

Table 4. Sequential Execution Time for Poisson Distribution (in seconds)

\begin{tabular}{cccccccccccc}
\hline $\begin{array}{c}\text { Trials } \\
\mathbf{n}\end{array}$ & $\mathbf{1}$ & $\mathbf{2}$ & $\mathbf{3}$ & $\mathbf{4}$ & $\mathbf{5}$ & $\mathbf{6}$ & $\mathbf{7}$ & $\mathbf{8}$ & $\mathbf{9}$ & $\mathbf{1 0}$ & $\mathbf{A v g .}$ \\
\hline $\mathbf{2 2 0 0}$ & 0.014 & 0.014 & 0.015 & 0.014 & 0.015 & 0.017 & 0.015 & 0.014 & 0.014 & 0.014 & 0.0146 \\
$\mathbf{2 5 0 0}$ & 0.021 & 0.021 & 0.021 & 0.022 & 0.021 & 0.018 & 0.022 & 0.019 & 0.021 & 0.021 & 0.0207 \\
$\mathbf{2 8 0 0}$ & 0.028 & 0.026 & 0.027 & 0.022 & 0.026 & 0.026 & 0.026 & 0.026 & 0.026 & 0.027 & 0.0260 \\
$\mathbf{3 1 0 0}$ & 0.027 & 0.029 & 0.028 & 0.028 & 0.028 & 0.029 & 0.028 & 0.027 & 0.027 & 0.027 & 0.0278 \\
$\mathbf{3 4 0 0}$ & 0.035 & 0.033 & 0.035 & 0.034 & 0.033 & 0.038 & 0.035 & 0.034 & 0.035 & 0.033 & 0.0345 \\
$\mathbf{3 7 0 0}$ & 0.040 & 0.041 & 0.040 & 0.042 & 0.039 & 0.039 & 0.039 & 0.045 & 0.044 & 0.038 & 0.0407 \\
$\mathbf{4 0 0 0}$ & 0.049 & 0.049 & 0.051 & 0.045 & 0.050 & 0.048 & 0.046 & 0.059 & 0.048 & 0.046 & 0.0491 \\
$\mathbf{4 3 0 0}$ & 0.057 & 0.058 & 0.057 & 0.054 & 0.056 & 0.054 & 0.056 & 0.060 & 0.055 & 0.057 & 0.0564 \\
$\mathbf{4 6 0 0}$ & 0.061 & 0.080 & 0.071 & 0.074 & 0.063 & 0.062 & 0.062 & 0.065 & 0.064 & 0.062 & 0.0664 \\
$\mathbf{4 9 0 0}$ & 0.071 & 0.069 & 0.072 & 0.070 & 0.075 & 0.077 & 0.067 & 0.074 & 0.079 & 0.157 & 0.0811 \\
\hline
\end{tabular}

For residual versus fitted value plot analysis, we should check if the residuals should be scattered randomly about zero which is true for each probability distributions in our case.

Histogram shows the spread of residuals and suggests the skewness if there is long tails and an outlier if a bar far away from the other bars. We can observe the skewness in almost each distribution but we cannot confirm the outlier, so histogram is used with normal probability plot to confirm the outlier, as in our case Binomial distribution has an outlier. 


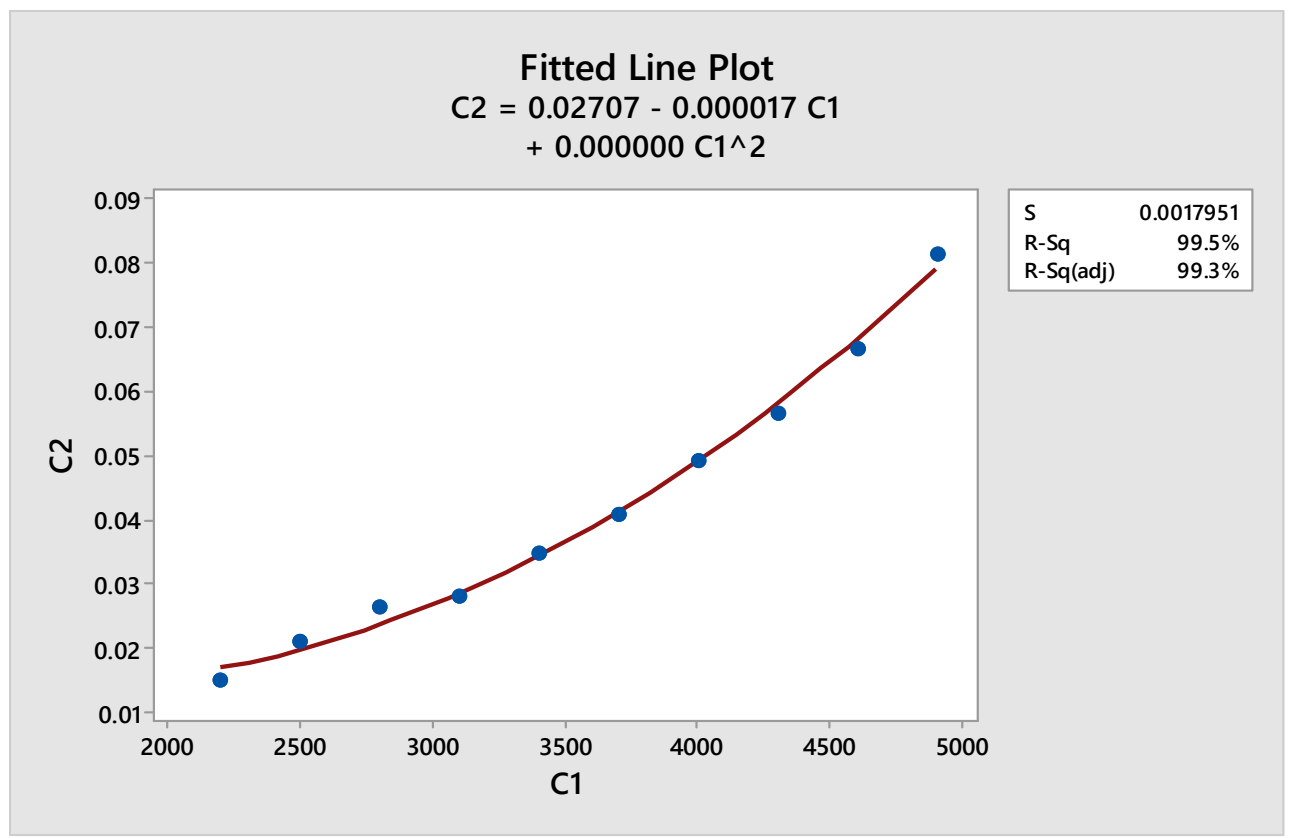

Figure 4a. Fitted line plot of Sequential Execution for Poisson Distribution.

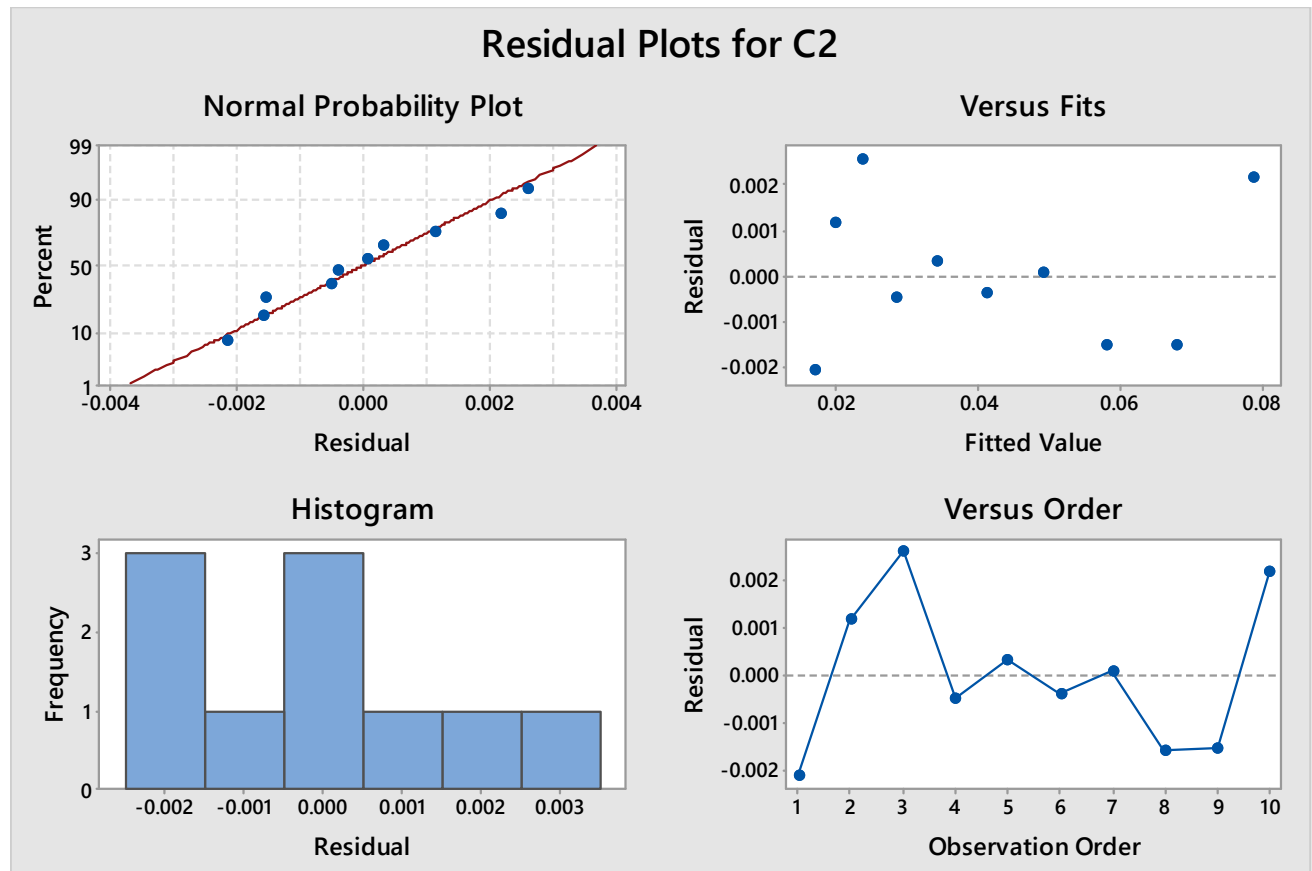

Figure 4b. Residual plot of Sequential Execution for Poisson Distribution.

In residuals versus observation order plot, the residuals in the plot should fluctuate in a random pattern around the central line which is true in our case for each distribution and we should also see if any correlation exists between the error terms that are near to each other which is not true in our case as no pattern is observed.

\subsection{For Parallel Execution}

For the results generated by Normal, Exponential, Binomial and Poisson Distributions refer to Tables 5, 6, 7 and 8 respectively.

For the corresponding fitted line plots refer to Figures 5a, 6a, 7a and 8a and for the corresponding residual plots refer to Figures 5b, 6b, 7b and $8 \mathrm{~b}$ respectively. 
Table 5. Parallel Execution Time for Normal Distribution (in seconds)

\begin{tabular}{ccccccccccccc}
\hline $\begin{array}{c}\text { Trials } \\
\mathbf{n}\end{array}$ & $\mathbf{1}$ & $\mathbf{2}$ & $\mathbf{3}$ & $\mathbf{4}$ & $\mathbf{5}$ & $\mathbf{6}$ & $\mathbf{7}$ & $\mathbf{8}$ & $\mathbf{9}$ & $\mathbf{1 0}$ & Avg. \\
\hline $\mathbf{2 2 0 0}$ & 0.0061 & 0.0060 & 0.0060 & 0.0075 & 0.0087 & 0.0061 & 0.0060 & 0.0060 & 0.0060 & 0.0062 & 0.0065 \\
$\mathbf{2 5 0 0}$ & 0.0076 & 0.0083 & 0.0096 & 0.0066 & 0.0078 & 0.0076 & 0.0078 & 0.0078 & 0.0076 & 0.0076 & 0.0078 \\
$\mathbf{2 8 0 0}$ & 0.0095 & 0.0095 & 0.0095 & 0.0095 & 0.0096 & 0.0095 & 0.0083 & 0.0103 & 0.0086 & 0.0087 & 0.0093 \\
$\mathbf{3 1 0 0}$ & 0.0102 & 0.0112 & 0.0101 & 0.0102 & 0.0103 & 0.0123 & 0.0140 & 0.0108 & 0.0131 & 0.0105 & 0.0113 \\
$\mathbf{3 4 0 0}$ & 0.0138 & 0.0140 & 0.0121 & 0.0130 & 0.0142 & 0.0138 & 0.0133 & 0.0144 & 0.0149 & 0.0143 & 0.0138 \\
$\mathbf{3 7 0 0}$ & 0.0154 & 0.0163 & 0.0161 & 0.0153 & 0.0176 & 0.0185 & 0.0160 & 0.0173 & 0.0226 & 0.0234 & 0.0179 \\
$\mathbf{4 0 0 0}$ & 0.0197 & 0.0195 & 0.0242 & 0.0177 & 0.0167 & 0.0197 & 0.0253 & 0.0169 & 0.0218 & 0.0184 & 0.0200 \\
$\mathbf{4 3 0 0}$ & 0.0234 & 0.0202 & 0.0218 & 0.0235 & 0.0213 & 0.0236 & 0.0229 & 0.0216 & 0.0260 & 0.0233 & 0.0227 \\
$\mathbf{4 6 0 0}$ & 0.0236 & 0.0273 & 0.0254 & 0.0275 & 0.0220 & 0.0224 & 0.0220 & 0.0221 & 0.0218 & 0.0221 & 0.0236 \\
$\mathbf{4 9 0 0}$ & 0.0249 & 0.0251 & 0.0251 & 0.0247 & 0.0255 & 0.0253 & 0.0254 & 0.0249 & 0.0256 & 0.0249 & 0.0251 \\
\hline
\end{tabular}

On analyzing the fitted line plots of parallel implementation we can certainly say that the observed data is perfectly fitting to the linear regression model as the value of R-squared and adjusted R-squared is above 95\% for each distribution with binomial distribution having the lowest value as 96.3\% (see Figure 7a) and Normal and Exponential distribution having the highest value as $98.3 \%$ (see Figure 5a and Figure 6a). We can also observe that the value of ' $\mathrm{S}$ ' is also very low each distribution.

On observing the Residual plots we can analyze from Normal probability plot and Histogram that there is outlier in Binomial and Poisson distributions (see Figures 7b and 8b) as there is a point far away from the tail and skewness is also present in every distribution.

For residual versus fitted value plot analysis we can see that the residuals are scattered randomly about zero which for each probability distributions.

In residuals versus observation order plot the residuals in the plot is also fluctuating in a random pattern around the center line for each distribution.

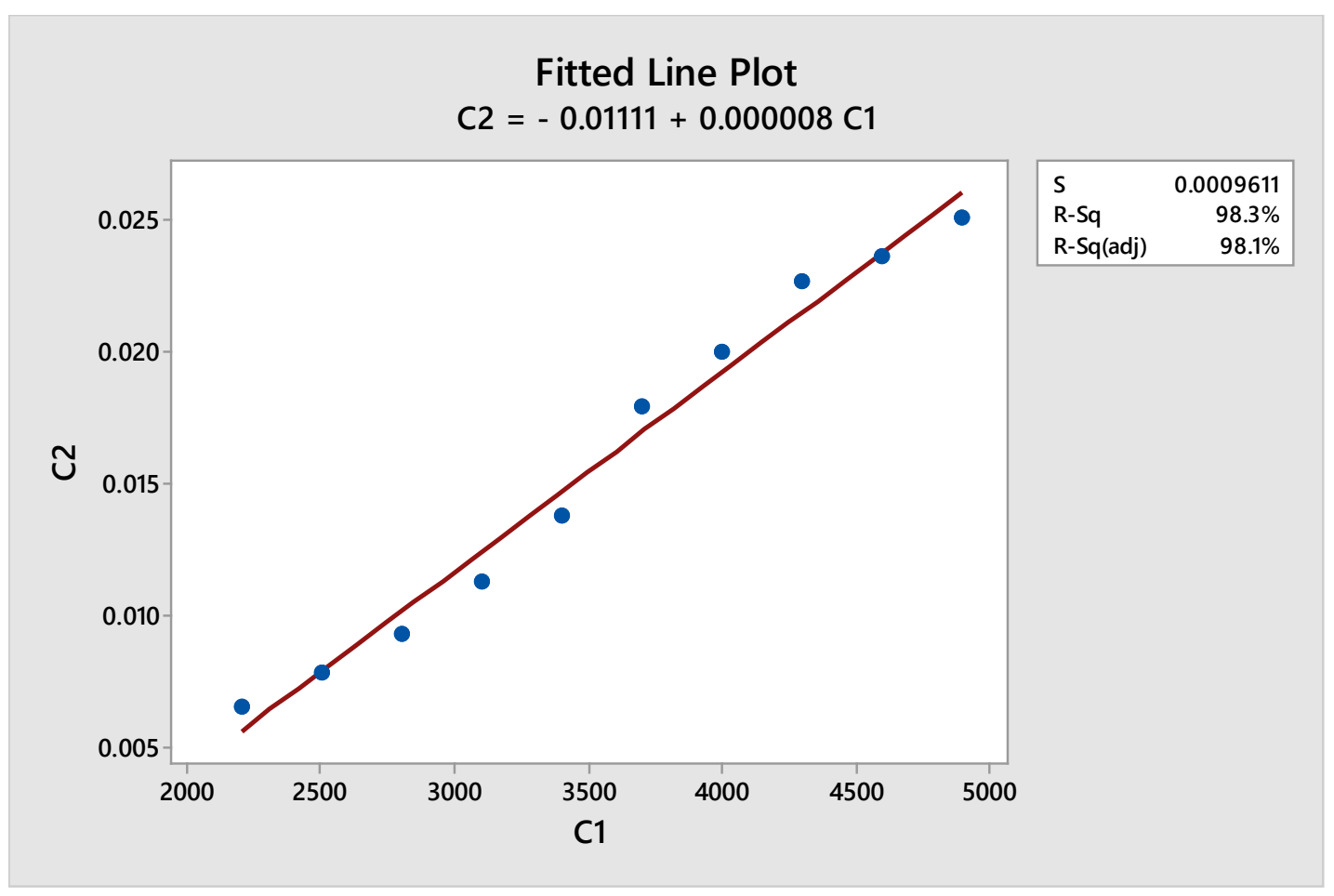

Figure 5a. Fitted line plot of Parallel Execution for Normal Distribution. 


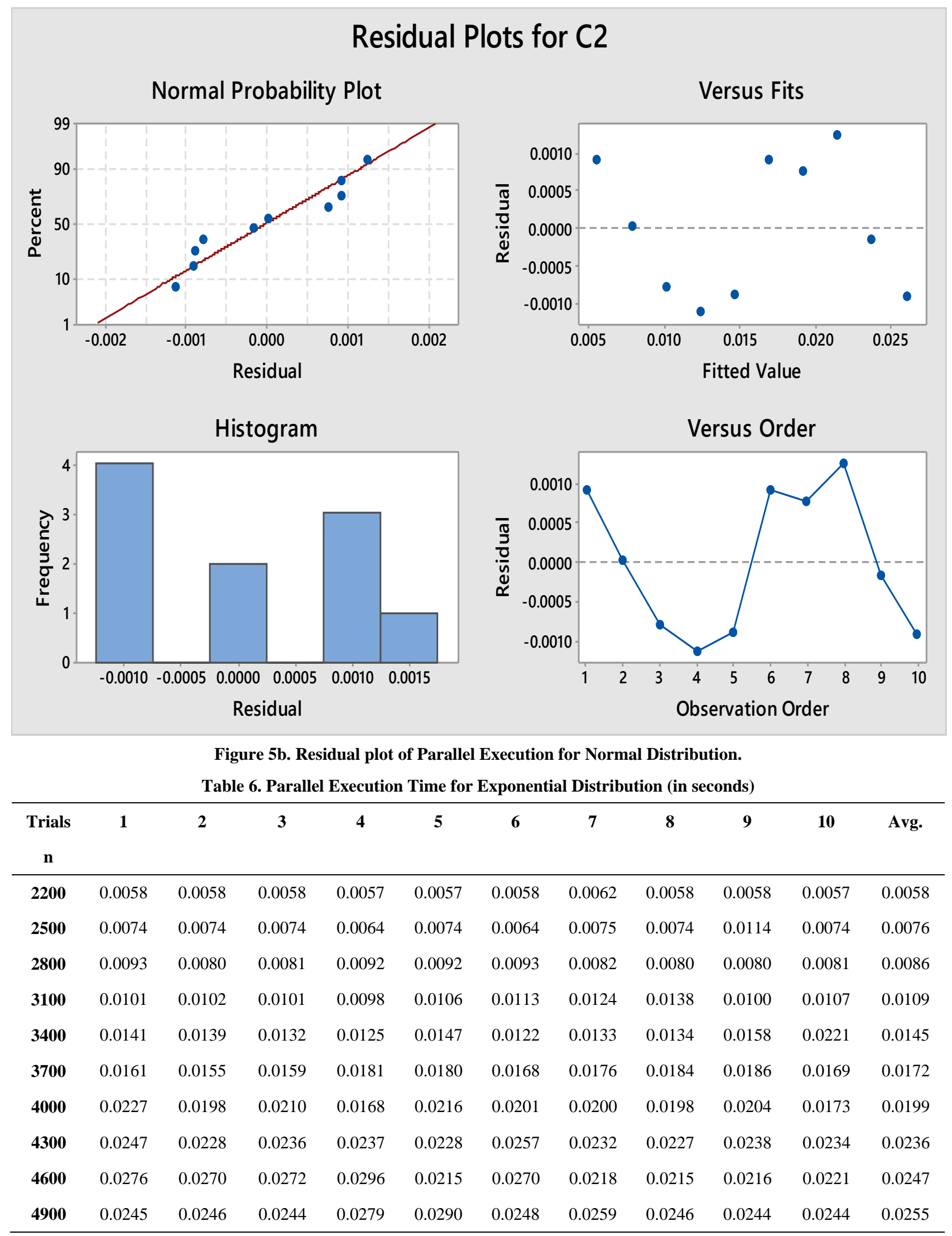


Table 7. Parallel Execution Time for Binomial Distribution (in seconds)

\begin{tabular}{cccccccccccc}
\hline $\begin{array}{c}\text { Trials } \\
\mathbf{n}\end{array}$ & $\mathbf{1}$ & $\mathbf{2}$ & $\mathbf{3}$ & $\mathbf{4}$ & $\mathbf{5}$ & $\mathbf{6}$ & $\mathbf{7}$ & $\mathbf{8}$ & $\mathbf{9}$ & $\mathbf{1 0}$ & Avg. \\
\hline $\mathbf{2 2 0 0}$ & 0.0050 & 0.0058 & 0.0058 & 0.0049 & 0.0057 & 0.0050 & 0.0057 & 0.0057 & 0.0057 & 0.0057 & 0.0055 \\
$\mathbf{2 5 0 0}$ & 0.0074 & 0.0074 & 0.0063 & 0.0066 & 0.0074 & 0.0074 & 0.0064 & 0.0074 & 0.0074 & 0.0073 & 0.0071 \\
$\mathbf{2 8 0 0}$ & 0.0094 & 0.0101 & 0.0080 & 0.0093 & 0.0093 & 0.0080 & 0.0080 & 0.0081 & 0.0081 & 0.0084 & 0.0087 \\
$\mathbf{3 1 0 0}$ & 0.0106 & 0.0119 & 0.0099 & 0.0104 & 0.0105 & 0.0098 & 0.0108 & 0.0116 & 0.0101 & 0.0109 & 0.0107 \\
$\mathbf{3 4 0 0}$ & 0.0145 & 0.0136 & 0.0129 & 0.0149 & 0.0151 & 0.0149 & 0.0139 & 0.0147 & 0.0123 & 0.0150 & 0.0142 \\
$\mathbf{3 7 0 0}$ & 0.0170 & 0.0185 & 0.0182 & 0.0181 & 0.0182 & 0.0178 & 0.0171 & 0.0192 & 0.0178 & 0.0188 & 0.0181 \\
$\mathbf{4 0 0 0}$ & 0.0198 & 0.0235 & 0.0177 & 0.0202 & 0.0192 & 0.0218 & 0.0213 & 0.0220 & 0.0213 & 0.0203 & 0.0207 \\
$\mathbf{4 3 0 0}$ & 0.0212 & 0.0261 & 0.0253 & 0.0218 & 0.0240 & 0.0240 & 0.0241 & 0.0404 & 0.0249 & 0.0254 & 0.0257 \\
$\mathbf{4 6 0 0}$ & 0.0295 & 0.0263 & 0.0277 & 0.0287 & 0.0216 & 0.0216 & 0.0229 & 0.0215 & 0.0215 & 0.0216 & 0.0243 \\
$\mathbf{4 9 0 0}$ & 0.0246 & 0.0245 & 0.0251 & 0.0289 & 0.0274 & 0.0243 & 0.0243 & 0.0243 & 0.0247 & 0.0275 & 0.0256 \\
\hline
\end{tabular}

Table 8. Parallel Execution Time for Poisson Distribution (in seconds)

\begin{tabular}{ccccccccccccc}
\hline $\begin{array}{c}\text { Trials } \\
\mathbf{n}\end{array}$ & $\mathbf{1}$ & $\mathbf{2}$ & $\mathbf{3}$ & $\mathbf{4}$ & $\mathbf{5}$ & $\mathbf{6}$ & $\mathbf{7}$ & $\mathbf{8}$ & $\mathbf{9}$ & $\mathbf{1 0}$ & Avg. \\
\hline $\mathbf{2 2 0 0}$ & 0.0050 & 0.0057 & 0.0060 & 0.0057 & 0.0087 & 0.0052 & 0.0057 & 0.0057 & 0.0058 & 0.0050 & 0.0059 \\
$\mathbf{2 5 0 0}$ & 0.0074 & 0.0066 & 0.0067 & 0.0066 & 0.0078 & 0.0073 & 0.0074 & 0.0073 & 0.0074 & 0.0064 & 0.0071 \\
$\mathbf{2 8 0 0}$ & 0.0093 & 0.0084 & 0.0081 & 0.0083 & 0.0080 & 0.0080 & 0.0080 & 0.0081 & 0.0080 & 0.0087 & 0.0083 \\
$\mathbf{3 1 0 0}$ & 0.0113 & 0.0126 & 0.0105 & 0.0099 & 0.0115 & 0.0127 & 0.0124 & 0.0136 & 0.0129 & 0.0107 & 0.0118 \\
$\mathbf{3 4 0 0}$ & 0.0148 & 0.0142 & 0.0151 & 0.0135 & 0.0158 & 0.0133 & 0.0149 & 0.0152 & 0.0149 & 0.0147 & 0.0146 \\
$\mathbf{3 7 0 0}$ & 0.0181 & 0.0178 & 0.0184 & 0.0191 & 0.0184 & 0.0176 & 0.0180 & 0.0174 & 0.0170 & 0.0163 & 0.0178 \\
$\mathbf{4 0 0 0}$ & 0.0212 & 0.0209 & 0.0188 & 0.0184 & 0.0200 & 0.0211 & 0.0201 & 0.0198 & 0.0180 & 0.0178 & 0.0196 \\
$\mathbf{4 3 0 0}$ & 0.0239 & 0.0228 & 0.0335 & 0.0246 & 0.0257 & 0.0274 & 0.0255 & 0.0224 & 0.0238 & 0.0221 & 0.0252 \\
$\mathbf{4 6 0 0}$ & 0.0288 & 0.0270 & 0.0269 & 0.0263 & 0.0215 & 0.0215 & 0.0214 & 0.0215 & 0.0215 & 0.0217 & 0.0238 \\
$\mathbf{4 9 0 0}$ & 0.0245 & 0.0281 & 0.0276 & 0.0281 & 0.0353 & 0.0245 & 0.0244 & 0.0260 & 0.0282 & 0.0279 & 0.0275 \\
\hline
\end{tabular}

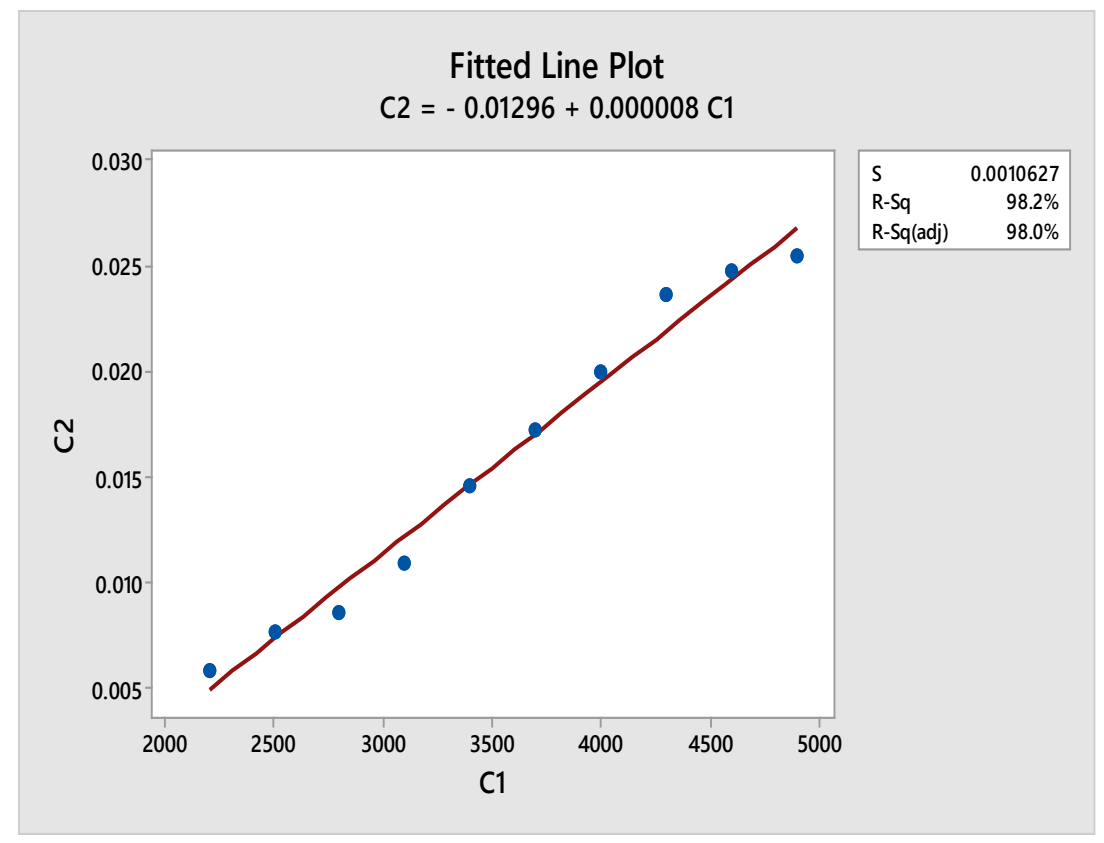

Figure 6a. Fitted line plot of Parallel Execution for Exponential Distribution. 


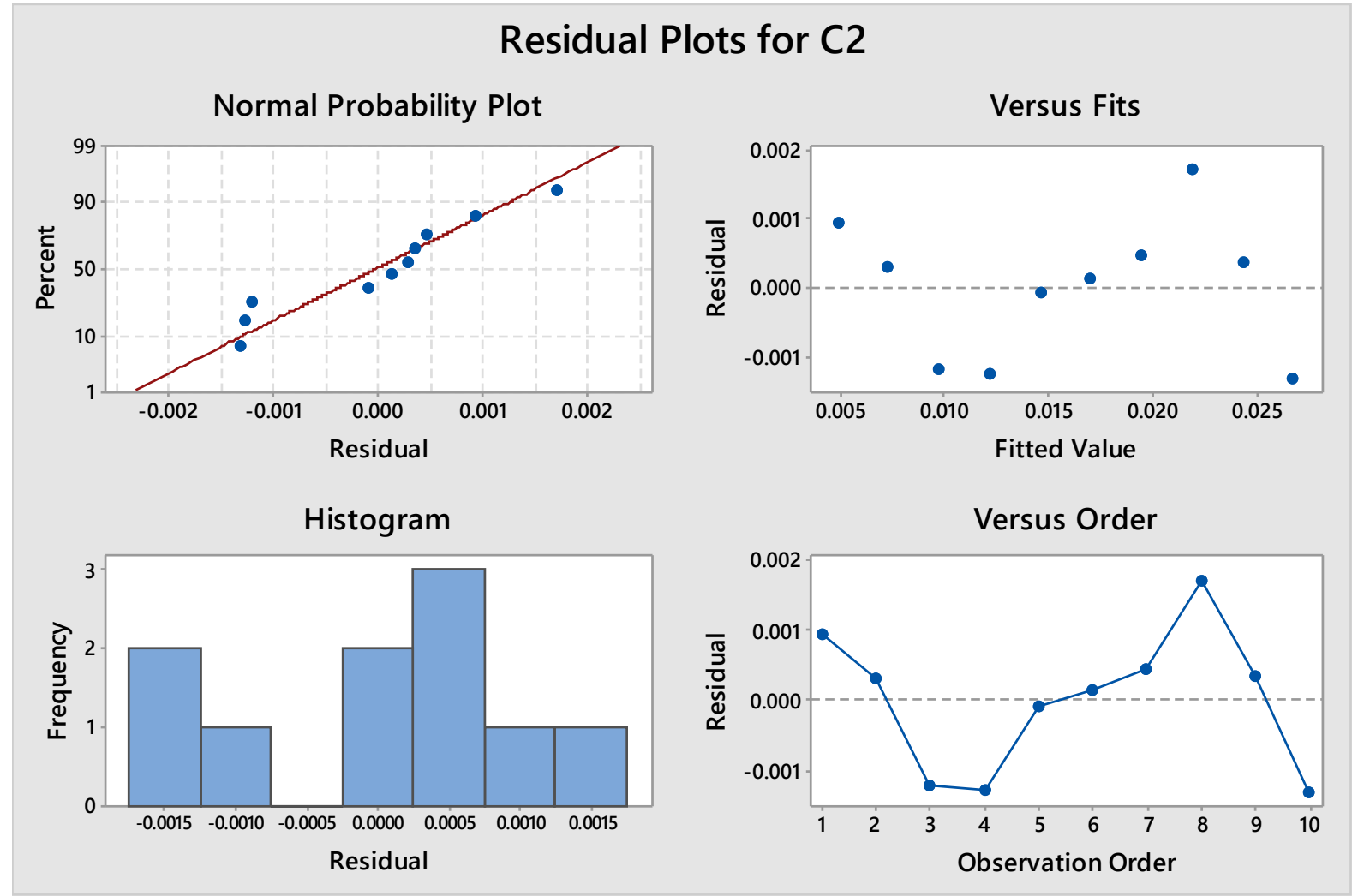

Figure 6b. Residual plot of Parallel Execution for Exponential Distribution.

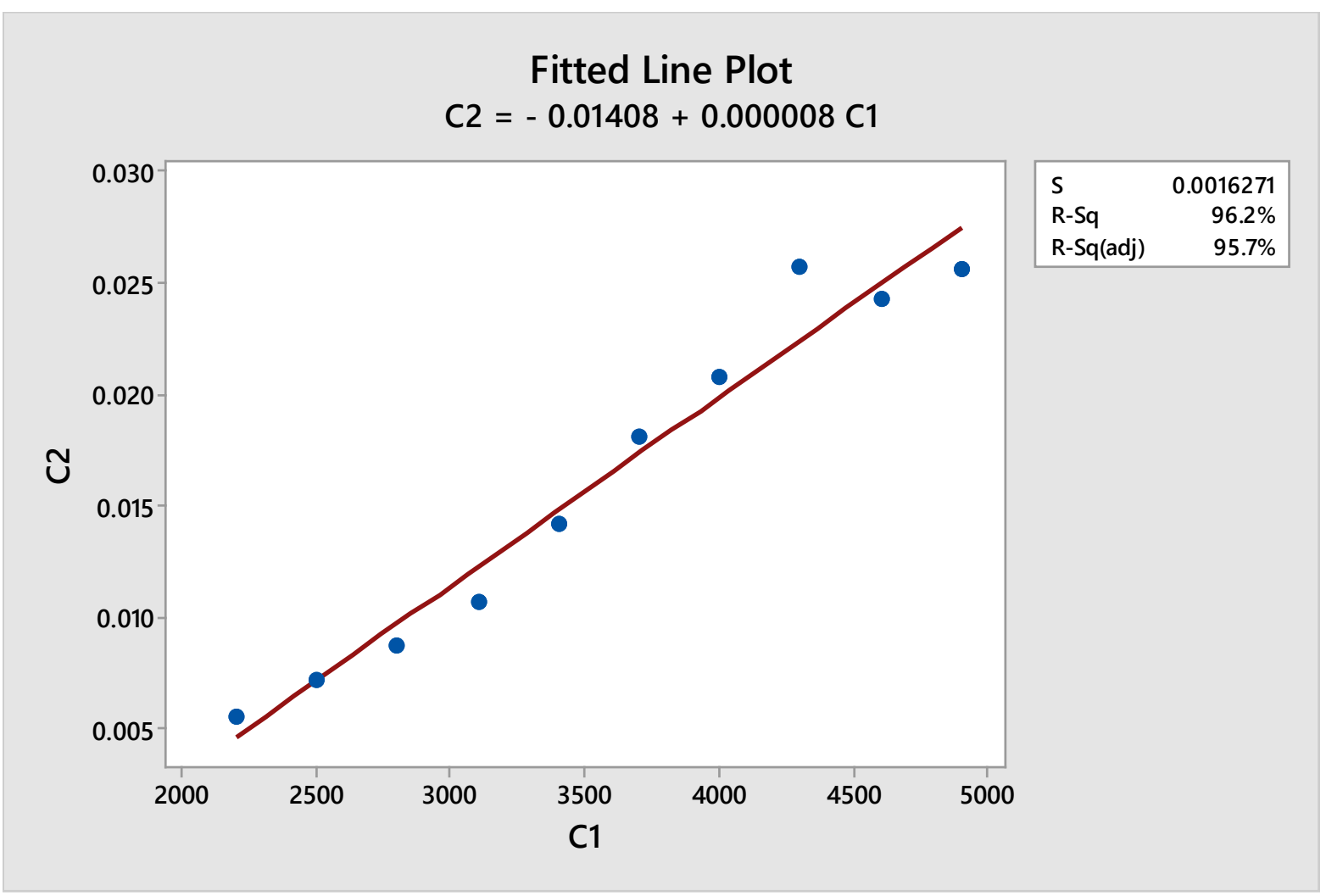

Figure 7a. Fitted line plot of Parallel Execution for Binomial Distribution. 


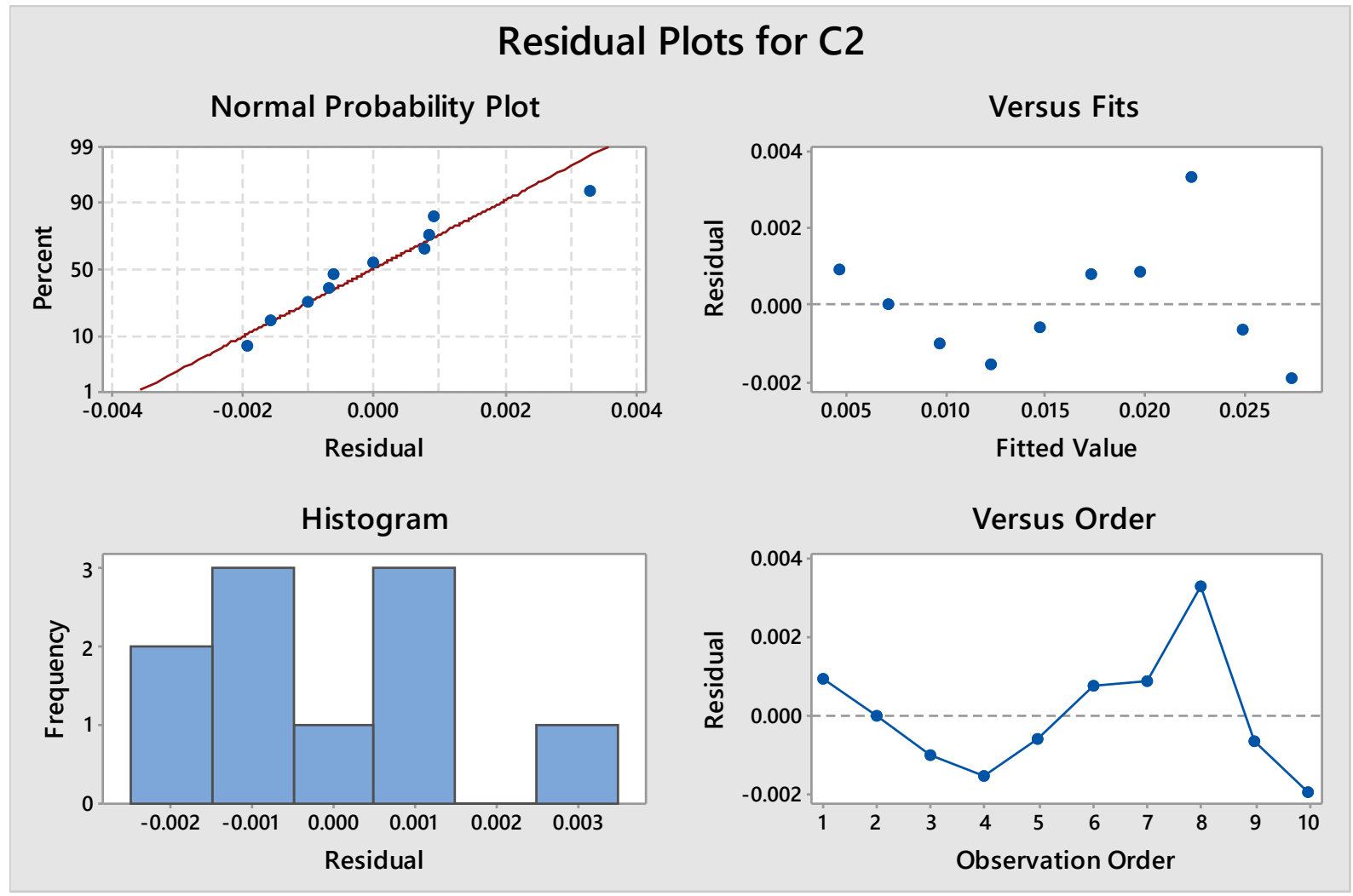

Figure 7b. Residual plot of Parallel Execution for Binomial Distribution.

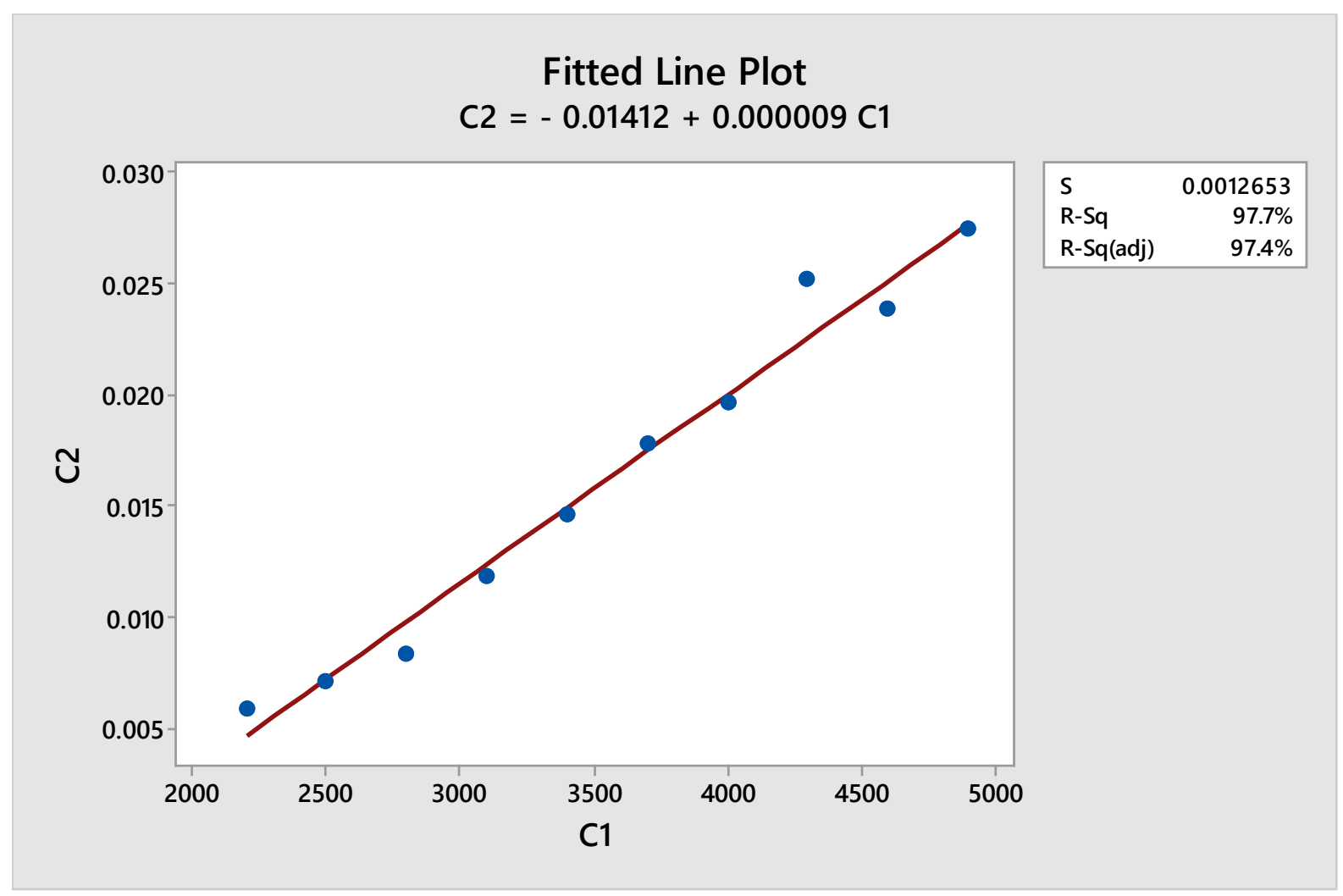

Figure 8a. Fitted line plot of Parallel Execution for Poisson Distribution. 


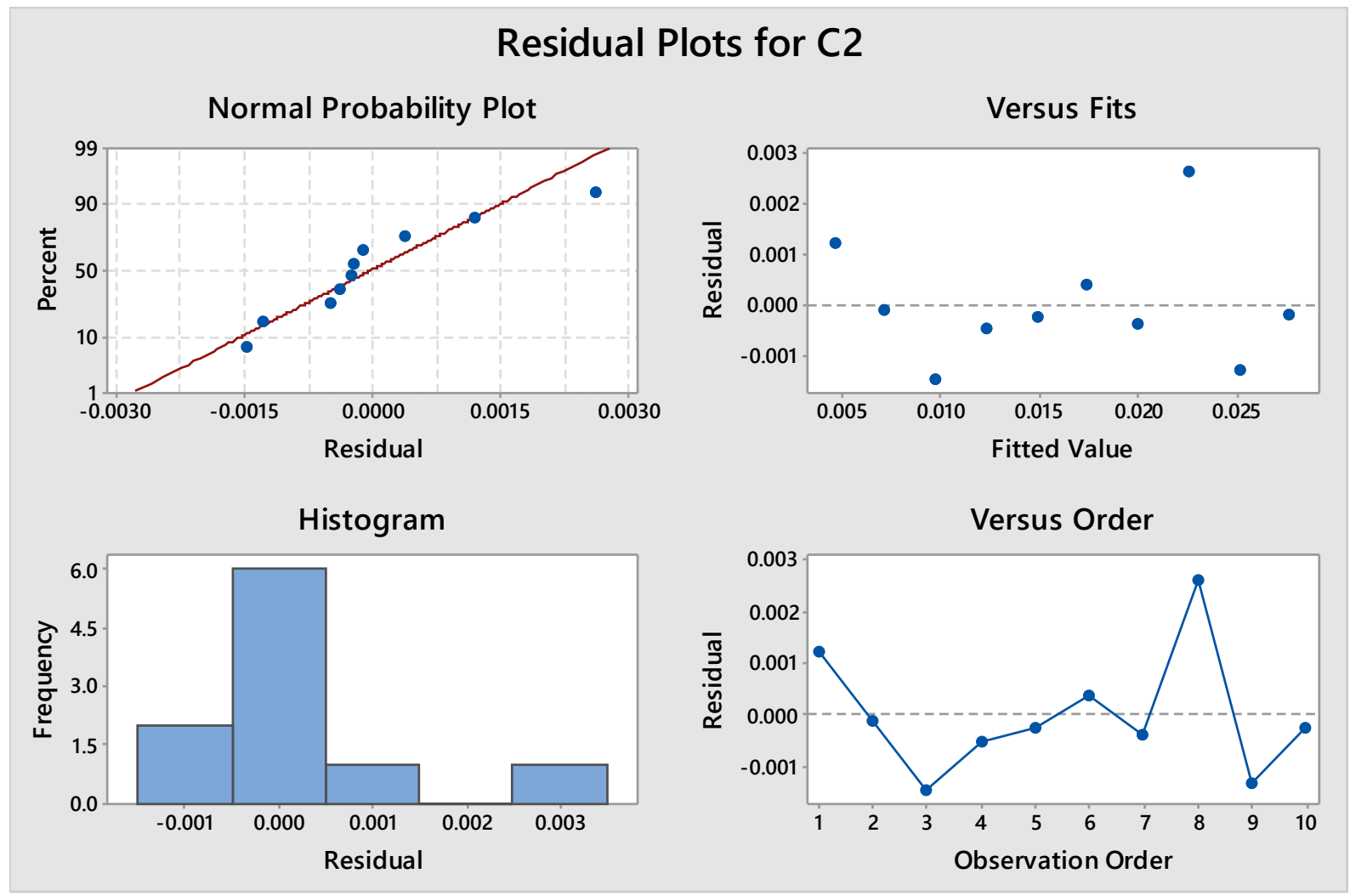

Figure 8b. Residual plot of Parallel Execution for Poisson Distribution.

As a final comment, Amir Schoor's algorithm, although initially developed for Sparse matrices, also performs well for dense matrices for two reasons. First, it is faster to work with rows only (as in Schoor's algorithm) than to work with both rows and columns (as in classical matrix multiplication). Secondly, as we have shown, the $\mathrm{n}^{2}$ comparisons turn out to be "heavier" (in terms of weight, with time of an operation being considered as its weight) than the $\mathrm{O}\left(\mathrm{d}_{1} \mathrm{~d}_{2} \mathrm{n}^{3}\right)$ multiplications on the average, wherein lies the concept of a weight based statistical bound whose empirical estimate over a finite feasible range is empirical $\mathrm{O}_{\text {emp }}\left(\mathrm{n}^{2}\right)$ in our case. Empirical O notation was introduced in [2].

Readers interested in recent works on fast matrix multiplication are referred to [11, 12, 13, 14].

\section{Conclusion}

From the observed data and plots we can certainly deduce that for parallel execution using the observed data is perfectly fitting the linear regression model when implemented in OpenMP using shared-memory architecture, which implies that we can certainly get an empirical O(n) average case time complexity for Amir Schoor's Algorithm for multiplication of two dense matrices while keeping pre-factor matrix as dense as triangular matrix and post-factor matrix fully dense.

Since the observed data from sequential execution is also perfectly fitting to the quadratic regression model, we can also confirm that an empirical $\mathbf{O}\left(\mathbf{n}^{2}\right)$ time complexity for average case can be obtained for sequential execution of matrix multiplication using Amir Schoor's Algorithm.

The robustness of the algorithm is totally ensured for both sequential and parallel implementations as the fitting remains the optimum even after subjecting the matrices elements over different input conditions using various probability distributions, which in turn supports the correctness of its empirical time complexity.

Since we are only dealing with rows of the matrices for performing matrix multiplication using Amir Schoor's algorithm we can easily say that our algorithm is not only fast for sparse matrices but also fast for dense matrices and is fast in both parallel and sequential environment as it is faster to work with rows only than both rows and columns.

\section{Ethical declaration}

The authors hereby declare that this research did not receive any funding and that they do not have any conflict of interest. 


\section{References}

[1] A. Schoor. (1982). “Fast Algorithm for Sparse Matrix Multiplication”. Information Processing Letters, 15(1982), pp. 87-89.

[2] S. Chakraborty and S. K. Sourabh. (2007). "On why an algorithmic time complexity measure can be system invariant rather than system independent”. Applied Mathematics and Computation, Volume 190, Issue 1, 1 July 2007, pp. 195-204.

[3] A. Kumari, N. K. Singh, and S. Chakraborty. (2015). "A Statistical Comperative Study of Some Sorting Algorithms”. International Journal in Foundations of Computer Science \& Technology (IJFCST),Vol. 5, No. 4, pp. 21-29, July 2015.

[4] S. K. Panigrahi, S. Chakraborty, and Jibitesh Mishra. (2012). "Statistical Bond of Bubble Sort Algorithm in Serial and Parallel Computations”. International Journal of Computer Science and Network (IJCSN), Volume 1, Issue 1, pp. 2277-5420, February 2012.

[5] https://www.minitab.com/en-us/support/documentation/.

[6] E. Horowitz, S. Sahni, and S. Rajsekaran. (2008). “Fundamentals of Computer Algorithms”, Universities Press, 2nd Edition, 2008, pp. 495-499.

[7] S. K. Panigrahi, Soubhik Chakraborty, Jibitesh Mishra. (2014). "Statistical analysis of Parallel Matrix Multiplication in SIMD model using 'p','p2','p3' processor's with different interconnection network”. 5th International Conference-Confluence The Next Generation Information Technology Summit (Confluence), IEEE Xplore, 978-1-4799-4236-7/14, pp. 858-865, September 2014.

[8] S. K. Panigrahi and S. Chakraborty. (2014). "Statistical Definition of an Algorithm in PRAM model \& Analysis of 2x2 Matrix Multiplication in 2n Processors Using Different Networks". IEEE International Advanced Computing Conference, 978-1-4799-2572, pp. 717-724, February 2014.

[9] S. Pacheco. (2011). “An Introduction to Parallel Programming”, Elsevier Inc., 2011.

[10] S. Aldea, A. Estebanez, et al. (2015). “An OpenMP Extension that Supports Thread-Level Speculation”, IEEE Transactions on Parallel and Distributed Systems, ISSN: 1045-9219, pp. 78-91, January 2015.

[11] G. Ballard, A. R. Benson, A. Druinsky, B. Lipshitz, and O. Schwartz. (2016). Improving the numerical stability of fast matrix multiplication. SIAM Journal on Matrix Analysis and Applications, 37(4), 1382-1418, 2016.

[12] G. Beniamini and O. Schwartz. (2019). Faster matrix multiplication via sparse decomposition. In Proceedings of the 31st ACM on Symposium on Parallelism in Algorithms and Architectures. ACM, 11-22, 2019.

[13] G. Bilardi and L. De Stefani. (2017). The I/O complexity of Strassen's matrix multiplication with recomputation. In Workshop on Algorithms and Data Structures. Springer, 181-192, 2017.

[14] E. Karstadt and O. Schwartz. (2020). Matrix Multiplication, A Little Faster, Journal of the ACM, Vol. 67, Issue 1, 1-31, April 2020. 